\title{
Corrigendum
}

\section{Mean-value theorems of multiplicative functions on additive arithmetic semigroups}

\section{Wen-Bin Zhang ${ }^{1,2}$}

1 Department of Mathematics, University of Illinois, 1409 West Green Street, Urbana, IL 61801, USA

2 Department of Mathematics, University of The West Indies, Mona, Kingston 7, Jamaica

Math. Z, 229, 195-233 (1998)

Received: 27 October 2003/Published online: 6 July 2004 - (C) Springer-Verlag 2004

It has been pointed out to me by R. Warlimont that the proof of Theorem (6.4.2) in [1] has a gap and the theorem is false. He has also constructed counter examples. In accordance, Theorem 1.6 in my paper [2] quoted in the title of this correction is false too. Actually, either of them requires more conditions on the multiplicative functions so that it can be true. In this correction we supply such a condition (see condition (2) below) and prove the following theorem.

Theorem. Suppose an additive arithmetic semigroup $\mathcal{G}$ satisfies the condition $(i)$ or (ii) in Theorem 1.6 in [2]. Let $f(a)$ be a multiplicative function such that $|f(a)| \leq 1$ for every $a \in \mathcal{G}$. If there exist a subset $\mathcal{P}_{1}$ of $\mathcal{P}$ and a constant $\epsilon>0$ such that

$$
\sum_{p \in \mathcal{P}_{1}} q^{-\partial(p)}<\infty
$$

and such that

$$
(\Re f(p))^{2}+(1+\epsilon)^{2}(\Im f(p))^{2} \leq 1
$$

and

$$
|\Im f(p)| \leq \frac{1}{\epsilon}(1-|\Re f(p)|)
$$

for all $p \notin \mathcal{P}_{1}$ then

$$
\lim _{m \rightarrow \infty} \frac{1}{G(m)} \sum_{\partial(a)=m} f(a) \quad \text { or } \quad \lim _{m \rightarrow \infty} \frac{(-1)^{m}}{G(m)} \sum_{\partial(a)=m} f(a)
$$

exists. 
Proof. If $m_{f}=0$ there is nothing to prove. Hence we may assume that $m_{f}=0$ is not true. By the last part of Theorem 1.5 in [2], there exists a real number $\alpha$ such that

$$
\sum_{p} q^{-\partial(p)}\left(1-\Re\left(f(p) q^{-i \partial(p) \alpha}\right)\right)
$$

converges and

$$
1+\sum_{k=1}^{\infty} q^{-k \partial(p)(1+i \alpha)} f\left(p^{k}\right) \neq 0
$$

for all $p \in \mathcal{P}$. Then, we can deduce that

$$
\sum_{p} q^{-\partial(p)}\left(1-\Re\left(f(p) q^{i \partial(p) \alpha}\right)\right)
$$

converges from (1), (2), and (3). By the last part of Theorem 1.5 in [2] again, we have

$$
1+\sum_{k=1}^{\infty} q^{-k \partial(p)(1-i \alpha)} f\left(p^{k}\right) \neq 0
$$

for all $p \in \mathcal{P}$.

Then, by Theorem 1.4 in [2], from (3) and (5) respectively, we have

$$
\begin{aligned}
F(m)=A q^{m(1+i \alpha)} m^{\tau-1} \prod_{\partial(p) \leq m}\left(1-q^{-\partial(p)}\right)\left(1+\sum_{k=1}^{\infty} q^{-k \partial(p)(1+i \alpha)} f\left(p^{k}\right)\right) \\
+o\left(q^{m} m^{\tau-1}\right)
\end{aligned}
$$

and

$$
\begin{aligned}
F(m)= & A q^{m(1-i \alpha)} m^{\tau-1} \prod_{\partial(p) \leq m}\left(1-q^{-\partial(p)}\right)\left(1+\sum_{k=1}^{\infty} q^{-k \partial(p)(1-i \alpha)} f\left(p^{k}\right)\right) \\
& +o\left(q^{m} m^{\tau-1}\right) .
\end{aligned}
$$

It follows that

$$
\begin{aligned}
& A q^{m(1+i \alpha)} m^{\tau-1} \exp \left\{-\sum_{\partial(p) \leq m} q^{-\partial(p)}\left(1-f(p) q^{-i \partial(p) \alpha}\right)\right\} F_{1}(m) \\
& =A q^{m(1-i \alpha)} m^{\tau-1} \exp \left\{-\sum_{\partial(p) \leq m} q^{-\partial(p)}\left(1-f(p) q^{i \partial(p) \alpha}\right)\right\} F_{2}(m) \\
& \quad+o\left(q^{m} m^{\tau-1}\right),
\end{aligned}
$$


where $F_{1}(m)$ and $F_{2}(m)$ have respective limits $F_{1}$ and $F_{2}$ as $m \rightarrow \infty$ and $F_{1}$ and $F_{2}$ are nonzero by (4) and (6). By (6), the first term on the right-hand side of (9) is nonzero. We divide both sides of (9) by this term and take limits as $m \rightarrow \infty$. The right-hand side has a limit 1 by the convergence of (5). Hence we arrive at

$$
\frac{F_{1}}{F_{2}} \lim _{m \rightarrow \infty} q^{2 i m \alpha} \exp \left\{\sum_{\partial(p) \leq m} q^{-\partial(p)} f(p)\left(q^{-i \partial(p) \alpha}-q^{i \partial(p) \alpha}\right)\right\}=1 .
$$

It follows that

$$
\sum_{p} q^{-\partial(p)}(\Im f(p)) \sin (\alpha \partial(p) \log q)
$$

converges. Also, that there exists a sequence $n_{m}, m=1,2, \ldots$ of integers such that

$$
\lim _{m \rightarrow \infty}\left(2 \alpha m \log q-2 \sum_{\partial(p) \leq m} q^{-\partial(p)}(\Re f(p)) \sin (\alpha \partial(p) \log q)+2 n_{m} \pi\right)
$$

exists.

From (3) and (10), we can conclude that

$$
\sum_{p} q^{-\partial(p)}(1-(\Re f(p)) \cos (\alpha \partial(p) \log q))
$$

converges (absolutely). Then it is easily to verify that the inequlity

$$
|(\Im f(p)) \cos (\alpha \partial(p) \log q)| \leq \frac{1}{\epsilon}(1-\Re f(p)) \cos (\alpha \partial(p) \log q)
$$

follows from (2). From (12) and (13), the (absolute) convergence of

$$
\sum_{p} q^{-\partial(p)}(\Im f(p)) \cos (\alpha \partial(p) \log q)
$$

follows.

Now, if $\alpha=0$, from (3) and (14),

$$
\sum_{p} q^{-\partial(p)}(1-f(p))
$$

converges. By the first part of Theorem 1.5 in [2], this implies, together with (4), that $f$ has a nonzero mean-value. If $\alpha \neq 0$, from (11),

$$
\alpha \log q-\sum_{\partial(p)=m} q^{-\partial(p)}(\Re f(p)) \sin (\alpha \partial(p) \log q)+\left(n_{m}-n_{m-1}\right) \pi \rightarrow 0
$$

as $m \rightarrow \infty$. Note that the modulus of the sum on the left-hand side is $\ll m^{-1} \rightarrow 0$ as $m \rightarrow \infty$. It follows, from (15), that $\alpha \log q=k \pi$ with some nonzero integer $k$ and $n_{m} \pi=m \alpha \log q+c$ with some constant $c$. Therefore, from (11),

$$
\sum_{p} q^{-\partial(p)}(\Re f(p)) \sin (\alpha \partial(p) \log q)
$$


converges. Then, from the convergence of (3), (14), and (16),

$$
\sum_{p} q^{-\partial(p)}\left(1-f(p) q^{-i \partial(p) \alpha}\right)
$$

converges. Let $g(a):=f(a) q^{-i \partial(a) \alpha}$. Then $g$ satisfies conditions (i) and (ii) of Theorem 1.5 in [2]. Thus the mean-value

$$
m_{g}=\lim _{m \rightarrow \infty} \frac{1}{G(m)} \sum_{\partial(a)=m} g(a)=\lim _{m \rightarrow \infty} \frac{q^{-i m \alpha}}{G(m)} \sum_{\partial(a)=m} f(a)
$$

exists and is nonzero. Then the existence of the alternative limits of the theorem follows according as $k$ is even or odd.

\section{References}

1. Knopfmacher, J., Zhang, W.-B.: Number Theory Arising From Finite Fields, anlytic and probabilistic theory, Monographs and textbooks in Pure and Applied Mathematics, 241. Marcel Dekker, New York-Basel, 2001

2. Zhang, W.-B.: Mean-value theorems of multiplicative functions on additive arithmetic semigroups. Math. Z. 229, 195-233 (1998) 